\title{
Capital social e fatores que influenciam o bem-estar dos agricultores familiares
}

\author{
Kátia Cristina Rodolpho Quedevez ${ }^{1}$ \\ Danilo Soares Monte-mor ${ }^{2}$
}

\begin{abstract}
Resumo
A agricultura familiar tem problemas associados ao bem-estar rural. A literatura científica apresenta elementos ligados aos cinco tipos de capital (econômico, humano, social, cultural e ambiental) como influenciadores na solução deste problema. Capital social é um conceito que tem sido explorado para mensurar o bem-estar em áreas rurais, mas há que se considerar os diferentes níveis de maturidade de capital social em que as comunidades se encontram. Esta pesquisa se propôs a identificar, à luz do conceito de capital social, fatores que influenciam a percepção de bem-estar de agricultores familiares em duas regiões agrícolas vizinhas e com diferentes níveis de maturidade de capital social, por meio de uma pesquisa exploratória quantitativa, com amostra de 417 agricultores. Os resultados apresentam evidências de que comunidades de agricultores familiares com nível mais baixo de amadurecimento de capital social consideram como entrave ao bem-estar rural o capital cultural. Comunidades com nível mais alto de amadurecimento de capital social percebem como entrave à sucessão familiar.
\end{abstract}

Palavras-chave: Agricultura familiar. Bem-estar rural. Capital social.

\begin{abstract}
Family farming has problems associated with rural well-being. The scientific literature presents elements related to the five types of capital (economic, human, social, cultural and environmental) as influencers in the solution of this problem. Social Capital is a concept that has been explored to measure well-being in rural areas, but we must consider the different levels of maturity of Social Capital in which the communities meet. This research aimed to identify, in light of the concept of Social Capital, factors that influence the perception of well-being of family farmers in two neighboring agricultural regions and with different levels of maturity of Social Capital, through a quantitative exploratory research, with a sample of 417 farmers. The results provide evidence that communities of family farmers with a lower level of social capital maturity consider the Cultural Capital as a barrier to rural well-being. Communities with a higher level of social capital maturity perceive Family Succession as an obstacle.
\end{abstract}

Keywords: Family Agriculture. Rural Well-Being. Share Capital.

\section{Introdução}

A agricultura continua a ser forte e, em alguns casos, crescente (INWOOD; SHARP, 2012), sustentando-se, principalmente, pelo ganho de produtividade decorrente da incorporação de tecnologias aos sistemas de produção e retornos de escala (LOWDER; SKOET; SINGH, 2014). Há que se considerar, no entanto, que áreas rurais têm características

\footnotetext{
${ }^{1}$ Mestre em Administração. katiaquedevez@gmail.com

2 Doutor em Administração e Ciências Contábeis. Professor da Fundação Instituto Capixaba de Pesquisas em Contabilidade, Economia e Finanças (FUCAPE Business School). danilo@fucape.b
} 
desafiadoras para o seu crescimento, como, por exemplo, distância física para acessar mercados, baixa massa crítica (TREGEAR; COOPER, 2016) e problemas associados ao bemestar dos produtores rurais (STIGLITZ; SEN; FITOUSSI, 2009).

Capital social é um conceito que tem sido explorado para mensurar bem-estar em áreas rurais (AGARWAL; RAHMAN; ERRINGTON, 2009), mas há que se considerar os diferentes níveis de maturidade de capital social em que as comunidades se encontram (PUTNAM, 2000). Uma vez que, individualmente, produtores rurais não costumam ter acesso às inovações tecnológicas e possuem baixas condições para atingir significativos ganhos de escala, elementos ligados ao conceito de capital social podem reduzir tal vulnerabilidade. "Entende-se por capital social as características da organização social, tais como redes, normas e confiança social, que facilitam a coordenação e a cooperação para benefício mútuo" (PUTNAM, 1995).

Diante deste cenário, o objetivo deste trabalho foi, à luz do conceito de capital social, identificar fatores associados à percepção de bem-estar de agricultores familiares. Para tanto, optou-se por utilizar nesta pesquisa uma comparação entre produtores rurais, de base familiar, localizados em duas regiões vizinhas, essencialmente agrícolas, com distintos níveis de amadurecimento de capital social. As regiões analisadas também apresentam distintos níveis de desenvolvimento econômico e social (IBGE, 2006) e adesão a cooperativas e associações rurais (INCAPER, 2013).

Os resultados obtidos sugerem que as variáveis educação/capacitação/treinamento, tecnologia e inovação, capital cultural, localização e sucessão familiar foram percebidas pelos agricultores como influenciadoras de bem-estar rural nas duas regiões pesquisadas. $\mathrm{Na}$ região de produtores rurais com nível mais baixo de amadurecimento de capital social, o capital cultural foi considerado como entrave ao bem-estar rural. E, na região de produtores rurais com nível mais alto de amadurecimento de capital social, a sucessão familiar foi considerada como entrave ao bem-estar rural.

O campo é vasto para novas pesquisas. A compreensão de como o nível de amadurecimento do capital social impacta no bem-estar rural pode contribuir na formulação de políticas públicas sociais que visem à promoção do bem-estar de agricultores familiares e colaborar, por exemplo, para a promoção de uma agenda cultural que estimule a economia em regiões rurais; a inserção de mulheres no campo e a redução do dilema da sucessão 
familiar nas propriedades rurais, em tempos em que o acesso à internet e a novas tecnologias estão impactando diretamente os processos de produção (UMANS; ARCE, 2014; LOWDER; SKOET; SINGH, 2014; STATHOPOULOU; PSALTOPOULOS; SKURAS, 2004).

\title{
2 Agricultura familiar, capital social e bem-estar rural
}

Apesar do poder econômico do agronegócio, a maioria das propriedades rurais ainda é explorada por agricultores familiares e, portanto, continua a ser importante entender a dinâmica familiar e seu desempenho (WHEELER et al., 2012). Estimativas da literatura agrícola dão conta de que existem pelo menos 570 milhões de explorações agrícolas em todo o mundo, das quais mais de 500 milhões podem ser consideradas de base familiar (LOWDER; SKOET; SINGH, 2014). Nota-se que, apesar das reduzidas áreas, razão pela qual, isoladamente, não produze expressivos volumes, a agricultura familiar possui alta relevância social e cultural.

A agricultura familiar possui relevada importância econômica no Brasil. Responde por "[...] mais de $40 \%$ do valor bruto da produção agropecuária e $74 \%$ da mão de obra nas propriedades rurais do país", segundo dados do Censo Agro (IBGE, 2006). O conceito de agricultura familiar é recorrente na legislação brasileira e é definido pelo IBGE (2006) como:

\begin{abstract}
Considera-se agricultor familiar e empreendedor familiar rural aquele que pratica atividades no meio rural, atendendo, simultaneamente, aos seguintes requisitos: I não detenha, a qualquer título, área maior do que 4 (quatro) módulos fiscais; II utilize predominantemente mão de obra da própria família nas atividades econômicas do seu estabelecimento ou empreendimento; III - tenha renda familiar predominantemente originada de atividades econômicas vinculadas ao próprio estabelecimento ou empreendimento; IV - dirija seu estabelecimento ou empreendimento com sua família.
\end{abstract}

\subsection{Capital social}

Após a década de 90, o conceito de capital social tem recebido destaque em uma série de estudos acadêmicos e debates teóricos como ferramenta para explicar e impulsionar o desenvolvimento econômico e social. No entanto, não existe uma definição acordada de capital social (TUNDUI; MACHA, 2014; MOYES et al., 2015; WOOLCOCK; NARAYAN, 2000; WOODHOUSE, 2006). Como resultado, sua definição permanece ambígua, disputada e vagamente compreendida (TUNDUI; MACHA, 2014). 
Do mesmo modo, não há consenso na literatura sobre como o capital social deveria ser medido (TUNDUI; MACHA, 2014). Fukuyama (1999) chega a afirmar que a maior falha do conceito de capital social é a falta de unanimidade na mensuração (GROOTAERT; VAN BASTELAER, 2002). Em essência, este problema surge devido ao fato de que o capital social é multidisciplinar, multidimensional e multifuncional, e por ter relação estreita com outras formas de capital, especialmente de capital humano (TUNDUI; MACHA, 2014).

$\mathrm{Na}$ busca pelo conceito de capital social e para ajudar a avaliar a sua utilidade para a teoria organizacional, Adler e Know (2002) sintetizaram a pesquisa teórica realizada em várias disciplinas e desenvolveram um quadro conceitual de capital social, apresentado no Quadro 1.

\section{Quadro 1 - Definições de Capital Social}

\begin{tabular}{|c|c|c|}
\hline \multicolumn{3}{|r|}{ Definições de Capital Social } \\
\hline $\begin{array}{l}\text { Externo } \\
\text { Versus } \\
\text { Interno } \\
\text { (Laços) }\end{array}$ & Autores & Definições de Capital Social \\
\hline \multirow{8}{*}{ Externo } & Baker & $\begin{array}{l}\text { "Um recurso que os atores derivam de estruturas sociais específicas e, em } \\
\text { seguida, usam para garantir seus interesses; criado por mudanças na } \\
\text { relação entre os atores" (1990: 6 19). }\end{array}$ \\
\hline & $\begin{array}{l}\text { Belliveau; } \\
\text { O'Reilly; } \\
\text { Wade }\end{array}$ & $\begin{array}{l}\text { "A rede pessoal de indivíduos e afiliações institucionais de elite" (1996: } \\
\text { 1572). }\end{array}$ \\
\hline & Bourdieu & $\begin{array}{l}\text { "O total dos recursos reais ou potenciais que estão ligados à posse de uma } \\
\text { rede durável de relações mais ou menos institucionalizadas de } \\
\text { conhecimento mútuo ou reconhecimento" (1986 2 48). }\end{array}$ \\
\hline & Bourdieu & $\begin{array}{l}\text { "Composta de obrigações sociais ('conexões'), que é conversível, em } \\
\text { determinadas condições, em capital econômico e pode ser } \\
\text { institucionalizado sob a forma de um título de nobreza" (1986: 243). }\end{array}$ \\
\hline & $\begin{array}{l}\text { Bourdieu; } \\
\text { Wacquant }\end{array}$ & $\begin{array}{l}\text { "A soma dos recursos, reais ou virtuais, que revertem para um indivíduo } \\
\text { ou um grupo, em virtude de possuir uma rede durável mais ou menos } \\
\text { institucionalizada, relações de conhecimento mútuo e reconhecimento" } \\
\text { (1992: 119). }\end{array}$ \\
\hline & $\begin{array}{l}\text { Boxman; } \\
\text { De Graaf; } \\
\text { Flap }\end{array}$ & $\begin{array}{l}\text { "O número de pessoas que podem ser esperadas para prestar apoio e os } \\
\text { recursos que essas pessoas têm à sua disposição" (1991: } 52 \text { 2). }\end{array}$ \\
\hline & Burt & $\begin{array}{l}\text { "[...] amigos, colegas e contatos mais gerais pelos quais recebem } \\
\text { oportunidades de usar o seu capital financeiro e humano" (1992: 9). }\end{array}$ \\
\hline & & "As oportunidades de relacionamentos em rede" (1997b: 3 55). \\
\hline
\end{tabular}

(Continua) 


\begin{tabular}{|c|c|c|}
\hline \multicolumn{3}{|r|}{ Definições de Capital Social } \\
\hline \multirow{10}{*}{$\begin{array}{l}\text { Externo } \\
\text { (cont.) }\end{array}$} & Knoke & $\begin{array}{l}\text { "O processo pelo qual os atores sociais criam e mobilizam suas conexões } \\
\text { de rede e entre as organizações para obter acesso aos recursos de outros } \\
\text { atores sociais" }(1999, \text { p. 18). }\end{array}$ \\
\hline & Portes & $\begin{array}{l}\text { "A capacidade dos atores para assegurar benefícios em virtude de } \\
\text { participação em redes sociais ou outras estruturas sociais" }(1998, \text { p. 6). }\end{array}$ \\
\hline & $\begin{array}{l}\text { Brehm; } \\
\text { Rahn } \\
\end{array}$ & $\begin{array}{l}\text { "A teia de relações de cooperação entre os cidadãos que facilitam a } \\
\text { resolução de problemas de ação coletiva" (1997, p. 999). }\end{array}$ \\
\hline & Coleman & $\begin{array}{l}\text { "O Capital Social é definido pela sua função. Não é uma simples entidade, } \\
\text { mas uma variedade de diferentes entidades que têm duas características } \\
\text { em comum: todos eles consistem em algum aspecto da estrutura social e } \\
\text { facilitam certas ações dos indivíduos que estão dentro da estrutura" (1990, } \\
\text { p. 302). }\end{array}$ \\
\hline & \multirow{2}{*}{ Fukuyama } & $\begin{array}{l}\text { "A capacidade das pessoas para trabalhar em conjunto, para fins comuns, } \\
\text { em grupos e organizações" }(1995, \text { p. 10). }\end{array}$ \\
\hline & & $\begin{array}{l}\text { "O Capital Social pode ser definido simplesmente como a existência de um } \\
\text { certo conjunto de valores informais ou normas compartilhadas entre os } \\
\text { membros de um grupo que permitem a cooperação entre eles" (1997). }\end{array}$ \\
\hline & Inglehart & $\begin{array}{l}\text { "Uma cultura de confiança e tolerância, em que surgem extensas redes de } \\
\text { associações voluntárias" (1997, p. 188). }\end{array}$ \\
\hline & $\begin{array}{l}\text { Portes; } \\
\text { Sensenbr } \\
\text { enner }\end{array}$ & $\begin{array}{l}\text { "Essas expectativas para a ação dentro de uma coletividade que afetam os } \\
\text { objetivos econômicos e objetivo, buscando comportamentos dos seus } \\
\text { membros, mesmo se essas expectativas não são orientadas para a esfera } \\
\text { econômica" (1993, p. 1323). }\end{array}$ \\
\hline & Putnam & $\begin{array}{l}\text { "Características da organização social, tais como redes, normas e } \\
\text { confiança social que facilitam a coordenação e a cooperação para } \\
\text { benefício mútuo" (199, p. } 67) \text {. }\end{array}$ \\
\hline & Thomas & $\begin{array}{l}\text { "Esses meios e processos voluntários desenvolvidos dentro da sociedade } \\
\text { civil que promovem desenvolvimento para todo o conjunto" (1996, p. 11). }\end{array}$ \\
\hline \multirow{5}{*}{ Ambos } & Laury & $\begin{array}{l}\text { "Ocorrência natural das relações sociais entre as pessoas que promovem } \\
\text { ou assistem a aquisição de habilidades e traços valorizados no mercado; } \\
\text { um ativo que pode ser tão significativo como legados financeiros em } \\
\text { contabilidade para a manutenção da desigualdade em nossa sociedade" } \\
(1992, \text { p. 100). }\end{array}$ \\
\hline & $\begin{array}{l}\text { Nahapiet; } \\
\text { Ghoshal }\end{array}$ & $\begin{array}{l}\text { "A soma dos recursos reais e potenciais disponíveis presentes } \\
\text { internamente e derivados a partir da teia de relações possuídas por uma } \\
\text { unidade individual ou social.; compreende tanto a rede e os ativos que } \\
\text { podem ser mobilizados através dela" (1998, p. } 243) \text {. }\end{array}$ \\
\hline & Pennar & $\begin{array}{l}\text { "A teia de relações sociais que influencia o comportamento individual que } \\
\text { afeta o crescimento econômico" }(1997, \text { p. 154). }\end{array}$ \\
\hline & Schiff & $\begin{array}{l}\text { "O conjunto de elementos da estrutura social que afeta as relações entre } \\
\text { as pessoas e são entradas ou argumentos da função de produção e / ou } \\
\text { utilidade" (1992, p. 160). }\end{array}$ \\
\hline & Woolcock & $\begin{array}{l}\text { "Informação, confiança e normas de reciprocidade inerentes à sua rede } \\
\text { social" (1998, p. 153). }\end{array}$ \\
\hline
\end{tabular}

Fonte: Adler e Know (2002). 


\subsection{Bem-estar rural}

As graves crises financeiras internacionais, a partir do final do século XX, colocaram em xeque algumas das principais economias do planeta e estimulam uma reflexão sobre o modelo de desenvolvimento adotado pela maioria dos países. Percebe-se que, por mais importante que sejam o crescimento econômico e o acúmulo de riquezas pelas nações, elas não podem sobrepor-se ao bem-estar das pessoas (STIGLITZ; SEM; FITOUSSI, 2009).

Stiglitz, Sem e Fitoussi (2009) defendem que, para entender o conceito de bem-estar, é necessário recorrer a uma definição multidimensional, a partir de pesquisas mundiais, onde foram identificadas dimensões que devem ser observadas e apreendidas simultaneamente:

I. As condições de vida materiais (rendimento, consumo e riqueza); II. A saúde; III. A educação; IV. As atividades pessoais, entre elas o trabalho; V. A participação na vida política e na governança; VI. Os laços e relações sociais; VII. O meio ambiente (situação presente e futura); VIII. A insegurança, tanto econômica quanto física. Todas essas dimensões modelam o bem-estar de cada um; entretanto, muitas delas são ignoradas pelas ferramentas tradicionais de medição dos rendimentos.

Indicadores econômicos e sociais como o Produto Interno Bruto (PIB) e o Índice de Desenvolvimento Humano (IDH), ainda os mais utilizados, são importantes ferramentas para a elaboração de políticas públicas para o desenvolvimento e impactam na definição das estratégias das organizações. Aprimorá-los é, sem dúvida, uma medida que traz avanços no planejamento das ações dos setores público e privado (STIGLITZ; SEM; FITOUSSI, 2009).

Bates (2009), em seu estudo, descreve mais um indicador, proposto em 1972, o da Felicidade Interna Bruta (FIB), pelo rei butanês Jigme Singya Wangchuck. Sen (2000) procurou demonstrar na sua obra que o desenvolvimento pode ser visto como um processo de expansão das liberdades reais.

Mesmo sendo vasto o número de estudos científicos sobre indicadores de desempenho em empresas, não há consenso sobre eles (KOTANE; KUZMINA-MERLINO, 2012; COSTA, 2013). A avaliação do desempenho de pequenas empresas, por exemplo, inclui indicadores financeiros e não financeiros (KOTANE; KUZMINA-MERLINO, 2012). Se, no caso das micro e pequenas empresas, há ausência de medidas objetivas, em propriedades rurais de base familiar, a falta de consenso é ainda mais latente (COSTA, 2013), o que inviabilizaria uma pesquisa do gênero. Escalas de desempenho autopercebido são utilizadas para desempenharem tal função (COVIN; SLEVIN, 1989), assim como utilizadas neste estudo. 
Portanto, como uma vasta literatura tem encontrado no conceito de capital social elementos para a superação de obstáculos, ganhos de produtividade, desenvolvimento local e bem-estar de produtores rurais, os quais se alinham ao objetivo deste trabalho, cujo desenho foi construído, como apresentado na Figura 1, a partir de alguns destes elementos: cooperação; redes sociais; confiança institucional; confiança governamental; confiança familiar e social; civismo, religiosidade, patriotismo e cidadania; infraestrutura, localização, tecnologia e inovação; capital cultural; capital ambiental; mulheres no campo; juventude rural e sucessão familiar.

Quadro 2 - Referências de elementos de capital social

\begin{tabular}{|c|c|}
\hline $\begin{array}{l}\text { Elementos de Capital Social } \\
\text { (Base do Construto) }\end{array}$ & Referências de cada elemento \\
\hline Cooperação & $\begin{array}{l}\text { TSAI; GOSHAL, 1998; WOOLCOCK, 2000; SORENSEN, 2000; } \\
\text { WOODHOUSE, 2006; TREGEAR; COOPER, } 2016\end{array}$ \\
\hline Redes Sociais & $\begin{array}{l}\text { COLEMAN, 1990; WOOLCOCK, 2000; PUTNAM; LEONARDI; } \\
\text { NANETTI, 1993; PUTNAM, 2000; WIESINGER, 2007; MARTELETO, } \\
\text { 2004; BEUGELSDIJK, 2009; TSAI; GOSHAL, 1998; MAMUN et al., } \\
\text { 2016; 2015; NAHAPIET; GHOSHAL, 1997; YOKOYAMA; SEKIGUCHI, } \\
2014\end{array}$ \\
\hline Confiança Institucional & $\begin{array}{l}\text { COLEMAN, 1990; PAXTON, 1999; KIM, 2005; WIGGINS; IRSTEN; } \\
\text { LLAMBÍ, } 2010\end{array}$ \\
\hline Confiança Governamental & $\begin{array}{l}\text { WIGGINS; KIRSTEN; LLAMBÍ, 2010; BOIX; POSNER, 1998; DELLER } \\
\text { et al., } 2010\end{array}$ \\
\hline Confiança Familiar e Social & $\begin{array}{l}\text { MONDELLI; KLEIN, 2014; TSAI; GHOSHAL, 1998; NAHAPIET; } \\
\text { GHOSHAL, 1998; NIEHM; SWINNEY; MILLER, 2008; SEGURA; } \\
\text { FORMIGONI, 2014; SANDERS; NEE, 1996; FITZGERALD et al., } 2010\end{array}$ \\
\hline $\begin{array}{l}\text { Civismo, Religiosidade, } \\
\text { Patriotismo e Cidadania }\end{array}$ & $\begin{array}{l}\text { PUTNAM, 1997; 2000; SVENDSEN; SORENSEN, 2006; WOOLCOCK; } \\
\text { NARAYAN, 2000; CROWE, 2006; WIESINGER, 2007; BESSER, 2009; } \\
\text { STOLLE; HOOGHE, 2005; SVENDSEN; SORENSEN, 2006; SORENSEN, } \\
\text { 2012; SVENDSEN; SORENSEN, 2012; WIESINGER, } 2007\end{array}$ \\
\hline $\begin{array}{l}\text { Infraestrutura, Localização, } \\
\text { Tecnologia e Inovação }\end{array}$ & $\begin{array}{l}\text { STATHOPOULOU; PSALTOPOULOS; PSALTOPOULOS, 2004; } \\
\text { WOODHOUSE, 2006; MARTELETO; SILVA, 2004; AGARWAL, 2010; } \\
\text { UMANS; ARCE, } 2014\end{array}$ \\
\hline Capital Cultural & AGARWAL, 2010; MATARASSO, 1999; BOURDIEU, 1996 \\
\hline Capital Ambiental & $\begin{array}{l}\text { WIESINGER, 2007; JONES; HALVADAKIS; SOPHOULIS, 2011; } \\
\text { MARSDEN; SMITH, 2005; NAHAPIET; GHOSHAL, } 1998\end{array}$ \\
\hline Mulheres no Campo & WIESINGER, 2007; AGARWAL; BINA, 2010 \\
\hline $\begin{array}{l}\text { Juventude Rural e Sucessão } \\
\text { Familiar }\end{array}$ & $\begin{array}{l}\text { KOUTSOU; PARTALIDOU; RAGKOS, 2014; INWOOD; CLARK, 2013; } \\
\text { INWOOD; SHARP, } 2012\end{array}$ \\
\hline
\end{tabular}

Fonte: Elaborado pelos autores. 


\section{Metodologia}

Para identificar os fatores associados ao bem-estar rural, segundo a percepção de agricultores familiares, à luz do conceito de capital social, foi adotada a metodologia quantitativa, de caráter descritivo e corte transversal. Para isso, optou-se pela coleta primária de dados, mediante a aplicação de um questionário estruturado em duas partes. A primeira, com 57 afirmações para cada variável do modelo, acompanhadas por uma escala de Likert de cinco níveis (variando de [1] discordo totalmente a [5] concordo totalmente). Desse modo, a nota final de cada construto foi formada pela média, por respondente, das variáveis de cada um dos elementos presentes na teoria do capital social. Na segunda parte do questionário, nove questões objetivas identificavam o perfil do agricultor familiar entrevistado (variáveis de controle). O questionário foi aplicado entre agricultores familiares por pesquisadores, no mês de dezembro de 2015 (HAIR; JOSEPH, 2005). Os agricultores familiares foram escolhidos de forma aleatória.

Tendo como base a literatura científica sobre elementos do capital social, este estudo propõe uma investigação exploratória sobre os fatores associados à percepção de melhoria do bem-estar de agricultores familiares (como descrito na Figura 1), de duas regiões vizinhas, essencialmente agrícolas, com diferentes níveis de amadurecimento de capital social.

As regiões analisadas também apresentam distintos níveis de desenvolvimento econômico e social (Quadro 3, inserido na próxima página) e adesão a cooperativas e associações rurais (INCAPER, 2013). 
Figura 1 - Desenho de Pesquisa

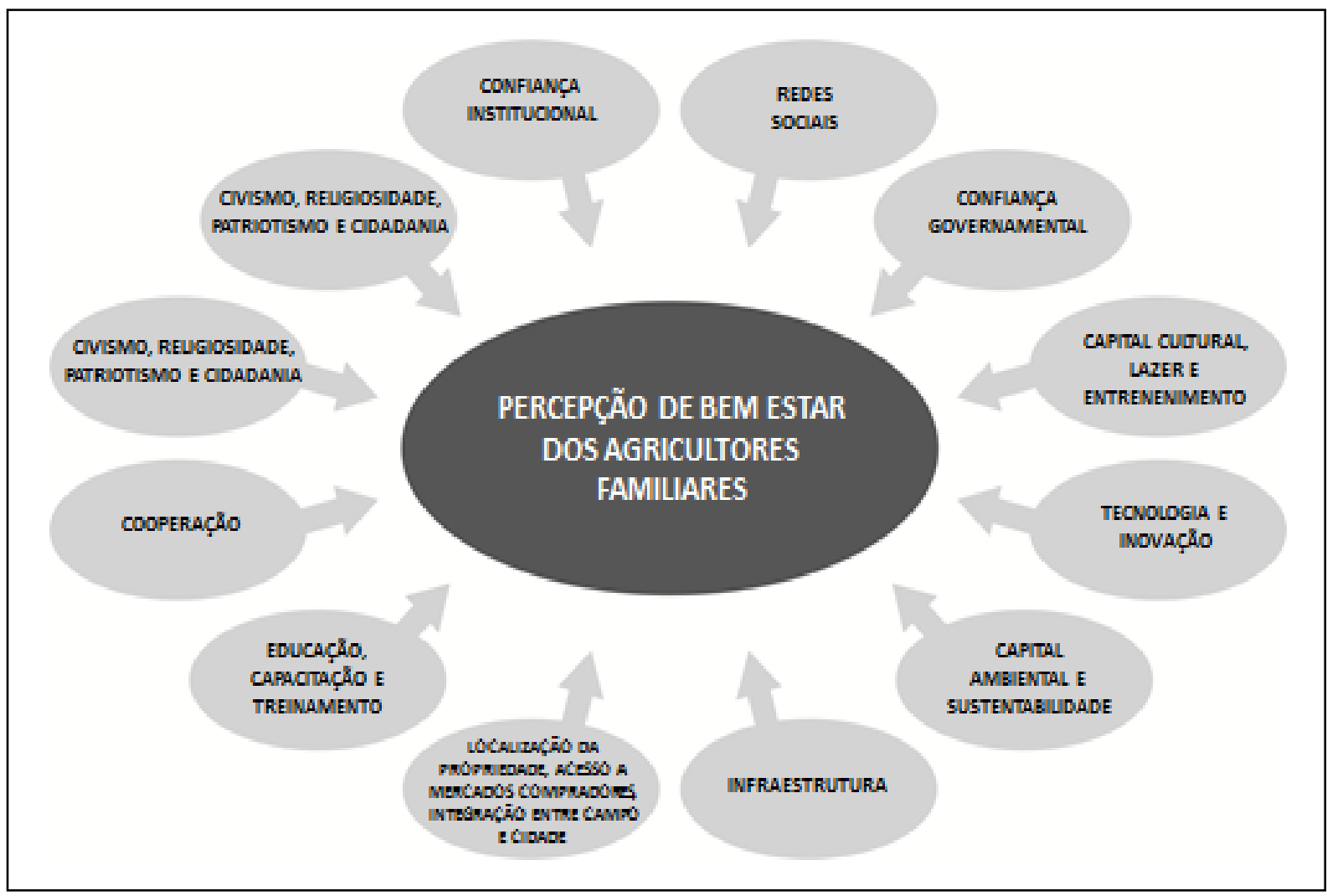

Fonte: Elaborado pelos autores.

Quadro 3 - Índices apurados entre os anos de 2008 a 2012 IDHM, PIB Per capita e renda per capita dos municípios

\begin{tabular}{|l|r|r|r|r|r|r|r|}
\hline Venda Nova do Imigrante \\
\hline Variáveis & Média & $\begin{array}{l}\text { Desvio- } \\
\text { Padrão }\end{array}$ & Mínimo & Q1 & Mediana & Q3 & Máximo \\
\hline População & 18.830 & 1.645 .76 & 16.165 & 17.437 & 19.216 & 20.023 & 21.094 \\
\hline IDHM & 0.67 & 0.03 & 0.65 & 0.65 & 0.65 & 0.65 & 0.73 \\
\hline $\begin{array}{l}\text { PIB } \\
\text { per capita }\end{array}$ & $9.361,75$ & $3.879,37$ & $4.448,65$ & $6.265,94$ & $8.445,40$ & $11.613,92$ & $15.141,74$ \\
\hline RENDA per capita & 625,34 & 73,72 & 586,55 & 586,55 & 586,55 & 586,55 & 756,66 \\
\hline Região do Caparaó Capixaba & \multicolumn{7}{|l|}{} \\
\hline Variáveis & Média & $\begin{array}{r}\text { Desvio } \\
\text { Padrão }\end{array}$ & Mínimo & Q1 & Mediana & Q3 & Máximo \\
\hline População & 16.317 & $8.774,55$ & 4.471 & 10.189 & 11.144 & 25.742 & 32.814 \\
\hline IDHM & 0.57 & 0.67 & 0.47 & 0.53 & 0.60 & 0.61 & 0.72 \\
\hline $\begin{array}{l}\text { PIB } \\
\text { per capita }\end{array}$ & $6.397,52$ & $2.683,736$ & $2.868,54$ & $4.318,84$ & $5.748,32$ & $8.252,63$ & $16.315,97$ \\
\hline RENDA per capita & 431,82 & 79,51 & 199,21 & 397,96 & 426,17 & 481,28 & 655,72 \\
\hline
\end{tabular}

Fonte: SEFAZ (2015). 
Após a coleta dos dados, as informações foram validadas segundo as variáveis de controle. Não foi verificada a existência de viés amostral e nem a presença de outliers. Na sequência das análises, foram estimados modelos de regressão linear. As variáveis explicativas utilizadas foram construídas a partir de alguns elementos da teoria do capital social: cooperação, redes sociais, educação/capacitação/treinamento, civismo/religiosidade, confiança institucional, capital cultural, confiança governamental, confiança familiar, infraestrutura, localização, tecnologia e inovação e capital ambiental. A seguir, o modelo de regressão utilizado:

Modelo: Percepção da melhoria do bem-estar rural $=B_{0}+\sum_{i=1}^{12} \beta_{i} X i+$ Controles $+E_{1}$, em que:

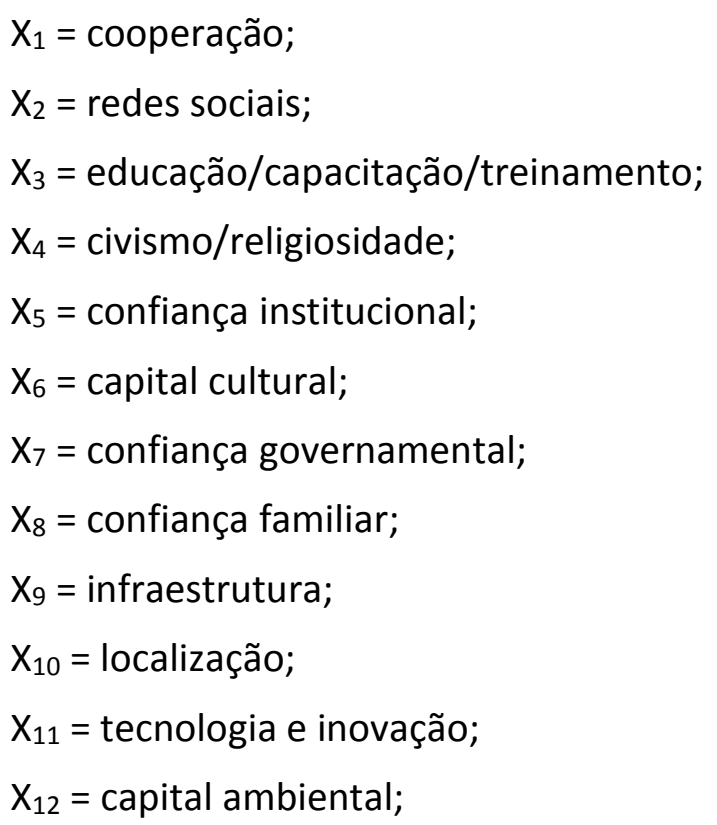

DCaparaó = 1 se Caparaó Capixaba (CAP); DVNI = 0 se Venda Nova do Imigrante (VNI);

Controles: gênero, idade, grau de escolaridade, maior grau de escolaridade do morador, números de filhos ou dependentes, experiência na atividade rural, horas semanais de dedicação à atividade, número de pessoas que trabalham na propriedade em tempo integral, número de pessoas que trabalham na propriedade em tempo parcial, mulheres no campo e sucessão familiar.

$E_{1}=$ termo de erro.

Para controle de elementos de capital social, foram inseridas na regressão dummies de gênero, idade, grau de escolaridade, maior grau de escolaridade do morador, números de filhos ou dependentes, experiência na atividade rural, horas semanais de dedicação à atividade, número de pessoas que trabalham na propriedade em tempo integral e número 
de pessoas que trabalham na propriedade em tempo parcial, mulheres no campo e sucessão familiar. Testes de interações foram realizados em cada grupo, condicionados pelas características de perfil, com o objetivo de verificar se as variáveis se comportavam diferentemente em cada perfil de grupo (HAIR; JOSEPH, 2005). O objetivo foi analisar, em cada núcleo de produtores, como cada variável impactava a percepção do agricultor familiar.

\section{Análise dos dados}

Neste estudo, foram utilizadas amostras de agricultores familiares do município de Venda Nova do Imigrante e da região do Caparaó Capixaba. A distribuição da amostra está descrita no Quadro 4.

\section{Quadro 4 - Características da amostra dos municípios (Venda Nova do Imigrante e Região} do Caparaó Capixaba)

\begin{tabular}{|c|c|c|c|c|}
\hline \multicolumn{3}{|c|}{ VENDA NOVA DO IMIGRANTE } & \multicolumn{2}{|c|}{ REGIÃO DO CAPARAÓ CAPIXABA } \\
\hline GÊNERO & № & $\%$ & № & $\%$ \\
\hline Masculino & 93 & $47,94 \%$ & 105 & $47,09 \%$ \\
\hline Feminino & 101 & $52,06 \%$ & 118 & $52,91 \%$ \\
\hline \multicolumn{5}{|l|}{ IDADE } \\
\hline Menos de 18 anos de idade & 2 & $1,03 \%$ & 5 & $2,24 \%$ \\
\hline Entre 18 e 25 anos de idade & 11 & $5,67 \%$ & 15 & $6,73 \%$ \\
\hline Entre 25 e 35 anos de idade & 37 & $19,07 \%$ & 28 & $12,56 \%$ \\
\hline Entre 35 e 45 anos de idade & 64 & $32,99 \%$ & 82 & $36,77 \%$ \\
\hline Acima de 50 anos de idade & 80 & $41,24 \%$ & 93 & $41,70 \%$ \\
\hline \multicolumn{5}{|c|}{ ESCOLARIDADE DO PROPRIETÁRIO RURAL } \\
\hline Analfabeto & 9 & $4,64 \%$ & 14 & $6,28 \%$ \\
\hline 1ㅇ grau incompleto & 98 & $50,52 \%$ & 115 & $51,57 \%$ \\
\hline 19 grau completo & 37 & $19,07 \%$ & 53 & $23,77 \%$ \\
\hline 20 grau incompleto & 22 & $11,34 \%$ & 22 & $9,86 \%$ \\
\hline 20 grau completo & 21 & $10,82 \%$ & 17 & $7,62 \%$ \\
\hline Superior incompleto & 3 & $1,55 \%$ & 2 & $0,90 \%$ \\
\hline Superior completo & 4 & $2,06 \%$ & & \\
\hline \multicolumn{5}{|c|}{ ESCOLARIDADE DO MORADOR DA PROPRIEDADE RURAL COM MAIOR NÍVEL DE ESCOLARIDADE } \\
\hline \multicolumn{5}{|l|}{\begin{tabular}{|l|} 
Analfabeto \\
\end{tabular}} \\
\hline 10 grau incompleto & 33 & $17,01 \%$ & 26 & $11,66 \%$ \\
\hline 10 grau completo & 22 & $11,34 \%$ & 21 & $9,42 \%$ \\
\hline 2ㅇ grau incompleto & 39 & $20,10 \%$ & 52 & $23,32 \%$ \\
\hline 2 grau completo & 43 & $22,16 \%$ & 65 & $29,15 \%$ \\
\hline Superior incompleto & 25 & $12,89 \%$ & 20 & $8,96 \%$ \\
\hline Superior completo & 29 & $14,95 \%$ & 38 & $17,04 \%$ \\
\hline
\end{tabular}




\begin{tabular}{|c|c|c|c|c|}
\hline \multirow{2}{*}{\multicolumn{3}{|c|}{\begin{tabular}{|l|} 
VENDA NOVA DO IMIGRANTE \\
NÚMERO DE FILHOS/DEPENDENTES
\end{tabular}}} & \multicolumn{2}{|c|}{ REGIÃO DO CAPARAÓ CAPIXABA } \\
\hline & & & & \\
\hline 1 & 47 & $24,23 \%$ & 30 & $13,45 \%$ \\
\hline 2 & 75 & $38,66 \%$ & 75 & $33,64 \%$ \\
\hline 3 & 41 & $21,13 \%$ & 62 & $27,80 \%$ \\
\hline 4 & 26 & $13,40 \%$ & 31 & $13,90 \%$ \\
\hline 5 & 1 & $0,52 \%$ & 20 & $8,97 \%$ \\
\hline 6 & 2 & $1,02 \%$ & 1 & $0,45 \%$ \\
\hline 7 & 1 & $0,52 \%$ & 2 & $0,89 \%$ \\
\hline 8 & 1 & $0,52 \%$ & 1 & $0,45 \%$ \\
\hline \multicolumn{5}{|c|}{ EXPERIÊNCIA NA ATIVIDADE RURAL } \\
\hline \multicolumn{5}{|l|}{ Menos de 1 ano } \\
\hline Entre 1 e 5 anos & 31 & $15,99 \%$ & 6 & $2,69 \%$ \\
\hline Entre 5 e 10 anos & 10 & $5,15 \%$ & 6 & $2,69 \%$ \\
\hline Entre 10 e 15 anos & 31 & $15,99 \%$ & 13 & $5,83 \%$ \\
\hline Mais de 20 anos & 122 & $62,87 \%$ & 198 & $88,79 \%$ \\
\hline \multicolumn{5}{|c|}{ HORAS SEMANAIS DEDICADAS À PROPRIEDADE } \\
\hline Abaixo de 1 hora/dia & & & 1 & $0,45 \%$ \\
\hline Entre 1 e 5 horas/dia & 33 & $17,01 \%$ & 30 & $13,45 \%$ \\
\hline Entre 5 e 10 horas/dia & 146 & $75,26 \%$ & 176 & $78,93 \%$ \\
\hline Acima de 10 horas/dia & 15 & $7,73 \%$ & 16 & $7,17 \%$ \\
\hline \multicolumn{5}{|c|}{$\begin{array}{l}\text { COLABORADORES QUE SE DEDICAM À PROPRIEDADE EM TEMPO INTEGRAL (8 horas diárias ou } \\
\text { mais, familiares como esposa e filhos, agregados e funcionários, todos registrados ou não) }\end{array}$} \\
\hline Não há colaboradores & 7 & $3,61 \%$ & 1 & $0,45 \%$ \\
\hline Entre 1 e 5 colaboradores & 157 & $80,93 \%$ & 188 & $84,30 \%$ \\
\hline Entre 6 e 15 colaboradores & 21 & $10,82 \%$ & 32 & $14,35 \%$ \\
\hline Entre 16 e 20 colaboradores & 8 & $4,12 \%$ & 1 & $0,45 \%$ \\
\hline Mais de 20 colaboradores & 1 & $0,52 \%$ & 1 & $0,45 \%$ \\
\hline \multicolumn{5}{|c|}{$\begin{array}{l}\text { COLABORADORES QUE SE DEDICAM À PROPRIEDADE EM TEMPO PARCIAL (menos de } 8 \text { horas } \\
\text { diárias e/ou eventuais, familiares como esposa e filhos, agregados e funcionários, todos } \\
\text { registrados ou não) }\end{array}$} \\
\hline Não há colaboradores & 10 & $5,15 \%$ & 61 & $27,35 \%$ \\
\hline Entre 1 e 5 colaboradores & 169 & $87,11 \%$ & 138 & $61,88 \%$ \\
\hline Entre 6 e 15 colaboradores & 12 & $6,19 \%$ & 22 & $9,87 \%$ \\
\hline Entre 16 e 20 colaboradores & 3 & $1,55 \%$ & 1 & $0,45 \%$ \\
\hline Mais de 20 colaboradores & & & 1 & $0,45 \%$ \\
\hline Número de observações & & 194 & & 223 \\
\hline Número Total de Observações & & & & 417 \\
\hline
\end{tabular}

Fonte: Dados coletados da pesquisa. Elaborado pela autora, com adaptações de Costa (2013).

A estatística descritiva de cada variável é apresentada com o objetivo de verificar como os dados se comportam em termos de posição e dispersão, proporcionando a verificação das congruências e diferenças entre as regiões analisadas (HAIR; JOSEPH, 2005), Venda Nova do Imigrante e região do Caparaó Capixaba. O teste t de diferença de médias foi 
utilizado para comparar as médias de cada variável com base nas respectivas amostras. Os resultados estão apresentados na Tabela 1.

\section{Tabela 1 - Estatística Descritiva}

\begin{tabular}{|c|c|c|c|c|c|c|}
\hline \multirow[t]{3}{*}{ Variáveis } & \multicolumn{2}{|c|}{$\begin{array}{c}\text { Venda Nova } \\
194 \text { observações }\end{array}$} & \multicolumn{2}{|c|}{$\begin{array}{l}\text { Caparaó Capixaba } \\
223 \text { observações }\end{array}$} & \multicolumn{2}{|c|}{ Dif. de Médias } \\
\hline & & Desvio & & Desvio & & \\
\hline & Média & Padrão & Média & Padrão & VNI-CAP & $P(|T|>|t|)$ \\
\hline Bem-Estar & 2,95 & 0,08 & 3,12 & 0,07 & $-0,16$ & 0,14 \\
\hline Redes Sociais & 4,32 & 0,09 & 4,49 & 0,07 & $-0,16$ & 0,16 \\
\hline Cooperação & 2,62 & 0,06 & 2,79 & 0,05 & $-0,17^{* *}$ & 0,03 \\
\hline Educação & 3,41 & 0,08 & 3,55 & 0,08 & $-0,14$ & 0,23 \\
\hline Civismo & 3,63 & 0,06 & 4 & 0,06 & $-0,37 * * *$ & 0 \\
\hline Confiança Institucional & 2,08 & 0,94 & 2,27 & 0,1 & $-0,18$ & 0,18 \\
\hline Capital Cultural & 1,94 & 0,1 & 2,33 & 0,11 & $-0,39 * *$ & 0,01 \\
\hline Confiança Governamental & 1,7 & 0,07 & 1,87 & 0,08 & $-0,17$ & 0,13 \\
\hline Confiança Familiar & 4,71 & 0,04 & 4,76 & 0,39 & $-0,04$ & 0,43 \\
\hline Infraestrutura & 4,24 & 0,04 & 4,07 & 0,04 & $0,16 * * *$ & 0 \\
\hline Localização & 4,34 & 0,05 & 3,75 & 0,07 & $0,59 * * *$ & 0 \\
\hline Tecnologia e Inovação & 3,53 & 0,09 & 3,23 & 0,02 & $0,29 * *$ & 0,03 \\
\hline Capital Ambiental & 4,32 & 0,03 & 4,23 & 0,03 & $0,09 *$ & 0,06 \\
\hline Mulheres no Campo & 4,68 & 0,07 & 4,64 & 0,06 & 0,03 & 0,68 \\
\hline Sucessão Familiar & 3,82 & 0,11 & 3,96 & 0,09 & $-0,13$ & 0,34 \\
\hline Gênero & 0,52 & 0,03 & 0,52 & 0,03 & 0 & 0,86 \\
\hline Idade & 4,07 & 0,06 & 4,08 & 0,06 & $-0,01$ & 0,89 \\
\hline Escol. Proprietário & 2,86 & 0,09 & 2,63 & 0,07 & $0,22 *$ & 0,05 \\
\hline Morador Maior Escol. & 4,53 & 0,12 & 4,67 & 0,1 & $-0,13$ & 0,38 \\
\hline Número de Filhos & 2,35 & 0,84 & 2,81 & 0,09 & $-0,46 * * *$ & 0 \\
\hline Tempo Atividade & 4,25 & 0,08 & 4,8 & 0,4 & $-0,54 * * *$ & 0 \\
\hline Horas Trab Semana & 2,9 & 0,03 & 2,92 & 0,03 & $-0,02$ & 0,65 \\
\hline Colaborador T. Integr & 2,17 & 0,04 & 2,16 & 0,02 & 0 & 0,85 \\
\hline Colaborador T. Parcial & 2,04 & 0,02 & 1,84 & 0,04 & $0,19 * * *$ & 0 \\
\hline
\end{tabular}

Fonte Dados coletados para a pesquisa aplicada. Elaborado pela autora.

$*, * * e^{* * *}$ representam coeficientes significativos a $10 \%, 5 \% 1 \%$, respectivamente.

\section{Discussão dos resultados}

Este estudo propôs uma investigação entre agricultores de base familiar em duas regiões vizinhas, essencialmente agrícolas, localizadas no Estado do Espírito Santo, com diferentes níveis de amadurecimento de capital social. As regiões analisadas também apresentam distintos níveis de desenvolvimento econômico e social (IBGE, 2006) e adesão a cooperativas e associações rurais (INCAPER, 2013). Foram entrevistados pessoalmente 417 
agricultores familiares em suas propriedades rurais. O método de avaliação utilizado foi o de regressões múltiplas.

Os resultados da estimação do Modelo, com e sem controle, estão descritos na Tabela 4, considerando-se, na parte superior da tabela, o efeito comum entre os agricultores familiares de Venda Nova do Imigrante e da região do Caparaó Capixaba; na segunda, a diferenciação entre Venda Nova e o Caparaó Capixaba, e, na terceira, as variáveis de controle da regressão. Evidencia-se que dos $99 \%$ de confiança, apenas a variável educação/capacitação/treinamento foi significativa nas amostras de agricultores familiares das duas regiões pesquisadas. Esse efeito não se mostrou estatisticamente diferente nas duas regiões. Nesse contexto, é possível afirmar que tanto os produtores respondentes de Venda Nova do Imigrante quanto os da região do Caparaó Capixaba perceberam que a variável educação/capacitação/treinamento tem efeito positivo na melhoria do bem-estar rural.

A constatação de que a variável educação/capacitação/treinamento obteve significância na amostra das duas regiões pode demonstrar que os agricultores familiares respondentes têm percebido o quanto o acesso ao uso de tecnologias e modernas práticas de gestão pode influenciar positivamente na melhoria do bem-estar familiar (STATHOPOULOU, PSALTOPOULOS; SKURAS, 2004), diante das características desafiadoras de crescimento das zonas rurais (TREGEAR; COOPER, 2016).

Com 95\% de confiança, capital cultural, tecnologia e inovação e sucessão familiar foram as variáveis que se mostraram significativas para explicar a percepção dos produtores nas regiões pesquisadas; e, com 90\% de confiança, a variável localização. Verificou-se que essas variáveis impactaram positivamente na percepção dos produtores, ou seja, quanto maior forem os valores atribuídos às variáveis capital cultural, tecnologia e inovação e localização, maior será a percepção dos agricultores de que essas variáveis impactem a melhoria do bem-estar familiar.

A variável capital cultural impactou positivamente no que se refere à percepção da melhoria do bem-estar de agricultores familiares de Venda Nova do Imigrante. Tal resultado pode estar alinhado aos estudos de Agarwal (2010), que sugere o capital cultural como uma dimensão explicativa de desempenho econômico rural e que o crescimento agrícola precisa de investimento substancial tanto em infraestrutura rural quanto em investigação de culturas (AGARWAL, 2010). 
O município de Venda Nova possui forte influência dos seus colonizadores europeus e mantém uma agenda fixa de eventos culturais tradicionais, típicos da cultura italiana, os quais envolvem a comunidade e movimentam o turismo o ano inteiro. A movimentação em torno desses eventos garante dividendos ao comércio, agroindústrias locais e impacta a economia do município, diretamente ligada ao agroturismo. Do contrário, o efeito da variável capital cultural impactou inversamente a percepção de melhoria do bem-estar dos proprietários rurais da região do Caparaó Capixaba.

Se em Venda Nova a forte influência da cultura italiana se reflete em ganhos para a economia local, pela realização, por exemplo, de tradicionais festas, eventos e festivais (PMVNI, 2016), no Caparaó, supõe-se que, devido à miscigenação dos colonizadores da região, não tenha ocorrido o mesmo, ou seja, a presença de uma agenda cultural tradicional que movimente a economia regional.

Observando-se que a variável tecnologia e inovação obteve, na amostra das duas regiões, uma significante percepção dos produtores sobre esse impacto positivo na melhoria do bem-estar familiar, este resultado corrobora o estudo de Umans e Arce (2014). Eles afirmam que a difusão da inovação é um dos aspectos que pode impactar positivamente o desempenho de agricultores familiares e que já existem relações obrigatórias, afetadas pelas transferências de capital e tecnologia (UMANS; ARCE, 2014).

A variável sucessão familiar foi estatisticamente significativa nas duas regiões para explicar a percepção dos produtores na melhoria do bem-estar familiar. Para agricultores da região do Caparaó Capixaba, o impacto foi positivo. Do contrário, a amostra de Venda Nova do Imigrante apresentou coeficiente inverso para a sucessão familiar, o que sugere que esses produtores consideram que sucessão familiar tem representado um entrave na melhoria do bem-estar (Tabela 2). 
Tabela 2 - Resultados da Regressão com Diferenciação Entre as Amostras de Venda Nova do Imigrante e Região do Caparaó Capixaba

\begin{tabular}{|c|c|c|c|c|}
\hline \multirow{2}{*}{ Qualidade Financeira } & \multicolumn{2}{|c|}{ Sem controle } & \multicolumn{2}{|c|}{ Com controle } \\
\hline & Coeficiente & $\mathbf{P}>|\mathbf{t}|$ & Coeficiente & $\mathbf{P}>|\mathbf{t}|$ \\
\hline Cooperação & 0,00 & 0,97 & 0,00 & 0,97 \\
\hline Redes Sociais & 0,08 & 0,28 & 0,91 & 0,25 \\
\hline Educação/Capacitação/Treinamento & 0,22 & 0,00 & 0,18 & 0,00 \\
\hline Civismo/Religiosidade & 0,11 & 0,20 & 0,11 & 0,21 \\
\hline Confiança Institucional & 0,55 & 0,44 & 0,02 & 0,73 \\
\hline Capital Cultural & 0,13 & 0,01 & 0,13 & 0,01 \\
\hline Confiança Governamental & $-0,02$ & 0,82 & 0,00 & 0,97 \\
\hline Confiança Familiar & 0,02 & 0,89 & 0,00 & 0,95 \\
\hline Infraestrutura & 0,16 & 0,21 & 0,10 & 0,41 \\
\hline Localização & 0,21 & 0,04 & 0,21 & 0,05 \\
\hline Tecnologia e Inovação & 0,14 & 0,01 & 0,12 & 0,04 \\
\hline Capital Ambiental & $-0,51$ & 0,73 & $-0,39$ & 0,79 \\
\hline Mulheres no Campo & $-0,80$ & 0,46 & $-0,11$ & 0,29 \\
\hline Sucessão Familiar & $-0,10$ & 0,04 & $-0,10$ & 0,04 \\
\hline INTCooperação & 0,04 & 0,78 & 0,01 & 0,90 \\
\hline INTRedes Sociais & 0,00 & 0,99 & $-0,04$ & 0,69 \\
\hline INTEducação/Capacitação/Treinamento & $-0,11$ & 0,23 & $-0,067$ & 0,48 \\
\hline INTCivismo/ Religiosidade & $-0,31$ & 0,79 & $-0,38$ & 0,75 \\
\hline INTConfiança Institucional & $-0,77$ & 0,39 & $-0,01$ & 0,83 \\
\hline INTCapital Cultural & $-0,18$ & 0,00 & $-0,19$ & 0,00 \\
\hline INTConfiança Governamental & 0,21 & 0,04 & 0,13 & 0,16 \\
\hline INTConfiança Familiar & 0,14 & 0,45 & 0,17 & 0,38 \\
\hline INTInfraestrutura & $-0,14$ & 0,35 & $-0,88$ & 0,58 \\
\hline INTLocalização & $-0,14$ & 0,28 & $-0,17$ & 0,20 \\
\hline INTTecnologia e Inovação & 0,31 & 0,70 & 0,02 & 0,74 \\
\hline INTCapital Ambiental & $-0,04$ & 0,82 & $-0,08$ & 0,63 \\
\hline INTMulheres no Campo & 0,14 & 0,26 & 0,17 & 0,17 \\
\hline INTSucessão Familiar & 0,16 & 0,02 & 0,16 & 0,02 \\
\hline Gênero & & & $-0,14$ & 0,18 \\
\hline Idade & & & 0,01 & 0,84 \\
\hline Escolaridade do Proprietário & & & 0,06 & 0,19 \\
\hline Morador com Maior Escolaridade & & & 0,48 & 0,19 \\
\hline Número de Filhos & & & $-0,46$ & 0,22 \\
\hline Tempo na Atividade Rural & & & 0,22 & 0,72 \\
\hline Horas de Trabalho por Semana & & & 0,19 & 0,86 \\
\hline Colaboradores em Tempo Integral & & & $-0,80$ & 0,54 \\
\hline Colaboradores em Tempo Parcial & & & 0,20 & 0,09 \\
\hline Número de Observações & & & & 417 \\
\hline
\end{tabular}

Fonte: Dados coletados para a pesquisa aplicada. Elaborado pela autora.

Inwood e Sharp (2012) afirmam que poucos estudos têm analisado diretamente fatores familiares, particularmente sucessão rural, podendo-se atribuir, inicialmente, a divergência percebida entre os resultados da amostra das duas regiões na variável sucessão familiar a dois fatores. 
Políticas públicas com foco no incentivo ao cultivo de café de qualidade superior (suporte governamental) foram implantadas na região do Caparaó no início do ano 2000 (INCAPER, 2013). Estas ações contribuíram para aumentar a competitividade do produto no mercado. No final do ano de 2015 , quando a coleta de dados foi realizada, o processo de implantação de incentivo ao cultivo de café de qualidade passava por um estágio de maturidade.

Se por um lado a crise política e econômica que atinge o Brasil tem causado alta taxa de desemprego dos municípios (MARTUSCELLI, 2013) e, por outro, a necessidade de manter mais pessoas envolvidas no trabalho na propriedade (INWOOD; SHARP, 2012), a permanência de jovens nas propriedades familiares garante-Ihes emprego e renda e pode estar proporcionando a sensação de segurança aos núcleos familiares da região do Caparaó.

De modo contrário, a amostra de Venda Nova do Imigrante apresentou coeficiente negativo para a variável sucessão familiar. Políticas públicas de estímulo ao cultivo de café de qualidade superior foram implantadas no município de Venda Nova do Imigrante no início dos anos 90 (INCAPER, 2013). É provável que essa geração de agricultores esteja passando por um processo de substituição de gerações, que poderia "[...] ser responsável para o declínio da coesão social e familiar" (PUTNAM, 2000; STOLLE; HOOGHE, 2005).

Apesar de vários estudos encontrarem no conceito de capital social evidências sobre desenvolvimento econômico regional, Putnam, Leonardi e Nanetti (1993); Putnam (1995; 2000); Svendsen e Sorensen (2006); Wiesinger (2007); Sorensen (2012); Tregear; Cooper (2016), seus resultados, de fato, não são unânimes.

O estudo de Svendsen e Sorensen (2006) mostrou a complexidade de se mensurar estatisticamente a influência de um capital intangível como o social. Tregear e Cooper (2016) alertam para que, no meio rural, a importância do engajamento de produtores rurais em ações coletivas, como grupos comunitários, associações ou redes, no desenvolvimento regional, não recebam interpretação restrita.

Outro trabalho, o de Sorensen (2012), testou a tese de Putnam (1997) de que engajamento cívico promove uma estrutura cooperativa e generalizada, e que isto leva à acumulação de capital social, sob a forma de redes inclusivas de cooperação, que são economicamente produtivas, e encontrou uma resposta negativa; portanto, o trabalho de 
Sorensen (2012) não forneceu nenhuma evidência de que maior densidade de associação está positivamente relacionada com os níveis mais elevados de desempenho econômico.

Wiesinger (2007) alerta que a era da globalização ou pós-modernidade, com a influência da televisão, da comunicação de massa e da internet, produziu nas áreas rurais um tipo de vizinhança virtual. Sua pesquisa não confirmou que o engajamento cívico, um dos pilares dos estudos de Putnam (1995; 1997), favoreça o desenvolvimento regional, porque as pessoas têm menos tempo para participar voluntariamente de associações locais (WIESINGER, 2007).

\section{Considerações finais}

Diante da relevância da agricultura familiar brasileira e pelos desafios enfrentados por produtores rurais, este estudo se propôs a identificar, à luz do conceito de capital social, fatores que influenciam a percepção de bem-estar de agricultores familiares, comparandoos em duas regiões agrícolas vizinhas, com diferentes níveis de amadurecimento de capital social. As duas regiões analisadas possuem características geográficas similares, apresentam distintos níveis de desenvolvimento econômico e social (IBGE, 2006) e adesão a cooperativas e associações rurais (INCAPER, 2013). Pela amplitude dos conceitos de capital social e bemestar rural, coube ao próprio pesquisador identificar variáveis que atendessem aos objetivos da pesquisa.

Se por um lado a agricultura tem relevante importância na economia do Brasil, sendo a responsável por vigorosos índices na pauta de exportações, por outro, produtores rurais enfrentam constantes desafios para o seu crescimento, como, por exemplo, a distância física para acessar mercados, a baixa massa crítica, a restrição ao crédito, as grandes jornadas de trabalho, a sazonalidade, a variação climática, a legislação ambiental. A literatura científica demonstra que capital social é um conceito que se tem mostrado eficiente na mensuração de bem-estar rural, mas devem ser considerados os diferentes níveis de maturidade desse capital em que as comunidades se encontram.

A pesquisa demonstrou que as variáveis tecnologia e inovação, capital cultural, educação/capacitação/treinamento, localização e sucessão familiar foram percebidas como influenciadoras de bem-estar rural pelos agricultores familiares de ambas as regiões 
analisadas, o que sugere que produtores rurais percebem que a educação impulsiona seu crescimento econômico e social. Particularmente, capital cultural e sucessão foram percebidas de maneira distinta nas duas regiões analisadas.

O capital cultural, percebido como influenciador de bem-estar pelos agricultores familiares de Venda Nova do Imigrante (município com alto nível de amadurecimento de capital social), pode estar ligado à dinâmica agenda anual de eventos tradicionais do município, referência de agroturismo no Brasil. Na região do Caparaó Capixaba (região com baixo nível de amadurecimento de capital social), a ausência de tal agenda pode ter colaborado na avaliação dos produtores, os quais perceberam o capital cultural como fator que impacta inversamente o bem-estar.

Sucessão familiar se configura um dilema na agricultura familiar e também foi percebida de maneira distinta pelos produtores rurais como influenciadora no bem-estar, sendo sua avaliação positiva na região do Caparaó Capixaba e inversamente negativa em Venda Nova do Imigrante. Ainda que considerada a distinção entre os cenários das duas regiões pesquisadas, o resultado pode demonstrar o atual dilema do agricultor familiar: preparar os filhos para a sucessão, ou incentivá-los a buscar novas atividades, em detrimento da propriedade rural.

Portanto, esta investigação apresenta algumas evidências: se considerada a similaridade das duas regiões agrícolas vizinhas e a distinção dos níveis de amadurecimento de capital social, as variáveis ligadas aos processos de produção e ao capital econômico (educação/capacitação/treinamento, tecnologia e inovação e localização), apresentaram convergência nos resultados; nos fatores ligados ao capital humano, os resultados foram distintos. Capital cultural e sucessão familiar apresentaram divergências entre as duas regiões. Em resumo: comunidades de agricultores familiares com nível mais baixo de amadurecimento de capital social consideram o capital cultural como entrave ao bem-estar rural. Comunidades com nível mais alto de amadurecimento de capital social percebem a sucessão familiar como entrave.

Os resultados sugerem que os desafios enfrentados por comunidades rurais com nível mais baixo de amadurecimento de capital social estão ligados ao acesso a uma agenda cultural dinâmica, que movimente a economia local. Comunidades com nível mais alto de 
amadurecimento de capital social carecem de transferência de conhecimento para gerações mais jovens.

O campo é vasto para novas pesquisas. A compreensão do impacto do nível de amadurecimento do capital social no bem-estar rural das comunidades pode contribuir na formulação de políticas públicas sociais, para promover melhor qualidade de vida aos agricultores familiares, colaborando, por exemplo, para a promoção de uma agenda cultural que estimule a economia em regiões rurais; a inserção de mulheres e jovens no campo e a redução do dilema da sucessão familiar nas propriedades rurais, em tempos em que o acesso à internet e a novas tecnologias está impactando diretamente os processos de produção (UMANS; ARCE, 2014; LOWDER; SKOET; SINGH, 2014; STATHOPOULOU; PSALTOPOULOS; SKURAS., 2004).

\section{Referências}

ADLER, P. S.; KWON, S. W. Social Capital: Prospects for a New Concept. Academy of Management Review, v. 27, n. 1, 2002. p. 17-40.

AGARWAL, B. Rethinking agricultural production collectivities. Economic and Political Weekly, p. 64-78, 2010.

AGARWAL, B.; RAHMAN, S.; ERRINGTON, A. Measuring the determinants of relative economic performance of rural areas. Journal of Rural Studies, v. 25, n. 3, p. 309-321, 2009.

BATES, W. Gross national happiness. Asian-Pacific Economic Literature, v. 23, n. 2, p. 1-16, 2009.

BESSER, T. L. Changes in small town social capital and civic engagement. Journal of Rural Studies, v. 25, n. 2, p. 185-193, 2009.

BEUGELSDIJK, S. A multilevel approach to social capital. International studies of management \& organization, v. 39, n. 2, p. 65-89, 2009.

BOIX, C.; POSNER, D. N. Social capital: Explaining its origins and effects on government performance. British journal of political science, v. 28, n. 4, p. 686-693, 1998.

BOURDIEU, P. The forms of capital. Cultural theory: An anthology, p. 81-93, 1986.

COLEMAN, J. S. Foundations of social theory. The Belknap Press of Harvard University Press. Cambridge and London, 1990. 
COSTA, T. A Relação Entre Capital Social e Desempenho Percebido em Micro e Pequenas Empresas. Dissertação (Mestrado) - FUCAPE Business School, Vitória, 2013.

COVIN, J. G.; SLEVIN, D. P. Strategic management of small firms in hostile and benign environments. Strategic management journal, v. 10, n. 1, p. 75-87, 1989.

CROWE, J. A. Community Economic Development Strategies in Rural Washington: Toward a Synthesis of Natural and Social Capital. Rural Sociology, v. 71, n. 4, p. 573-596, 2006.

DELLER, S. C. et al. The role of amenities and quality of life in rural economic growth. American Journal of Agricultural Economics, v. 83, n. 2, p. 352-365, 2010.

FITZGERALD, M. A. et al. Socially responsible processes of small family business owners: Exploratory evidence from the national family business survey. Journal of Small Business Management, v. 48, n. 4, p. 524-551, 2010.

FUKUYAMA, F. Social capital and the global economy. Foreign Affairs, v. 74, n. 5, p. 89-103, sep./oct. 1995.

GROOTAERT, C.; VAN BASTELAER, T. The role of social capital in development: An empirical assessment. Cambridge University Press, 2002.

HAIR, J.; JOSEPH F. Fundamentos de métodos de pesquisa em administração. Porto Alegre: Bookman, 2005.

IBGE. Censo Agropecuário: Agricultura Familiar: Primeiros Resultados 2006. Rio de Janeiro: Instituto Brasileiro de Geografia e Estatística - IBGE, 2006. Disponível em:

<http://www.ibge.gov.br/home/estatistica/economia/agropecuaria/censoagro/agri_famil iar_2006/familia_censoagro2006.pdf>. Acesso em: 20 out. 2015.

INCAPER. Relatórios do Programa de Assistência Técnica e Extensão Rural Proater 2011 2013. Espírito Santo: Instituto Capixaba de Pesquisa, Assistência Técnica e Extensão Rural. INCAPER, 2013. Disponível em:

<http://incaper.es.gov.br/media/incaper/proater/municipios>. Acesso em: 1 jul. 2016.

INWOOD, S. M.; SHARP, J. S. Farm persistence and adaptation at the rural-urban interface: Succession and farm adjustment. Journal of Rural Studies, v. 28, n. 1, p. 107-117, 2012.

JONES, N.; HALVADAKIS, C. P.; SOPHOULIS, C. M. Social capital and household solid waste management policies: a case study in Mytilene, Greece. Environmental Politics, v. 20, n. 2, p. 264-283, 2011.

KOTANE, I.; KUZMINA-MERLINO, I. Assessment of financial indicators for evaluation of business performance. European integration studies, n. 6, p. 216-224, 2012.

KOUTSOU, S.; PARTALIDOU, M.; RAGKOS, A. Young farmers' social capital in Greece: Trust levels and collective actions. Journal of Rural Studies, v. 34, p. 204-211, 2014. 
LOWDER, S. K.; SKOET, J.; SINGH, S. What do we really know about the number and distribution of farms and family farms in the world? Background paper for The State of Food and Agriculture, v. 8, 2014.

MAMUN, A. et al. Social capital and entrepreneurial competencies: A study among women micro-entrepreneurs in Malaysia. The Journal of Developing Areas, v. 50, n. 5, p. 363-370, 2016.

MARSDEN, T.; SMITH, E. Ecological entrepreneurship: sustainable development in local communities through quality food production and local branding. Geoforum, v. 36, n. 4, p. 440-451, 2005.

MARTELETO, R. M.; SILVA, A. B. de O. Redes e Capital Social: o enfoque da informação para o desenvolvimento local. Ciência da informação, v. 33, n. 3, p. 41-49, 2004.

MARTUSCELLI, D. E. et al. Crises políticas e capitalismo neoliberal no Brasil. 2013.

MATARASSO, F. Towards a local culture index. Measuring the cultural vitality of communities. Gloucester: Comedia, 1999.

MONDELLI, M. P.; KLEIN, P. G. Private equity and asset characteristics: the case of agricultural production. Managerial and Decision Economics, v. 35, n. 2, p. 145-160, 2014.

MOYES, D. et al. The stairway to Heaven? The effective use of social capital in new venture creation for a rural business. Journal of Rural Studies, v. 39, p. 11-21, 2015.

NAHAPIET, J.; GHOSHAL, S. Social capital, intellectual capital, and the organizational advantage. Academy of Management Review, v. 23, n. 2, p. 242-266, 1998.

NIEHM, L. S.; SWINNEY, J.; MILLER, N. J. Community Social Responsibility and Its Consequences for Family Business Performance. Journal of Small Business Management, v. 46, n. 3, p. 331-350, 2008.

PAXTON, P. Is social capital declining in the United States? A multiple indicator assessment 1. American Journal of Sociology, v. 105, n. 1, p. 88-127, 1999.

PMVNI. Prefeitura Municipal de Venda Nova do Imigrante. Disponível em: <vendanova.es.gov.br/website/site/Historico.aspx>. Acesso em: 2 nov. 2015.

PUTNAM, R. D. Bowling Alone: The collapse and revival of American community. New York: Simon \& Schuster, 2000.

. Bowling alone: America's declining social capital. Journal of democracy, v. 6, n. 1, p. 65-78, 1995.

Comunidade e democracia: a experiência da Itália moderna. Rio de Janeiro:

Fundação Getúlio Vargas, 1997. 
PUTNAM, R. D; LEONARDI, R.; NANETTI, R. Y. Making Democracy Work. In: RIDDELL, S.; TETT, L. (Eds.). Education, social justice and inter-agency working: Joined-up or fractured policy, 1993.

SANDERS, J. M.; NEE, V. Immigrant self-employment: The family as social capital and the value of human capital. American sociological review, p. 231-249, 1996.

SEFAZ. Índices dos Municípios. 2015. Disponível em:

<http://internet.sefaz.es.gov.br/informacoes/index.php>. Acesso em: 15 dez. 2015.

SEGURA, L. C.; FORMIGONI, H. Influence of Control and Family Management in the Indebtedness of Brazilian Open Business: a Quantitative Study. Brazilian Business Review, v. 11, n. 6, p. 50, 2014.

SEN, Amartya. Desenvolvimento como liberdade. Tradução: Laura Teixeira Motta; revisão técnica Ricardo Doninelli Mendes - São Paulo: Companhia das Letras, 2000

SORENSEN, C. Social capital and rural development: a discussion of issues. Social Capital Initiative Working Paper n. 10. 2000. The World Bank. Disponível em:

<http://Inweb18.worldbank.org/ESSD/sdvext.nsf/60ByDocName/PublicationsSocialCapitalSo cial-CapitallnitiativeWorkingPapers>. Acesso em: 15 dez. 2015.

SORENSEN, J. F. L. Testing the hypothesis of higher social capital in rural areas: the case of Denmark. Regional Studies, v. 46, n. 7, p. 873-891, 2012.

STATHOPOULOU, S.; PSALTOPOULOS, D.; SKURAS, D. Rural entrepreneurship in Europe: a research framework and agenda. International Journal of Entrepreneurial Behavior \& Research, v. 10, n. 6, p. 404-425, 2004.

STIGLITZ, J. E.; SEN, A. K.; FITOUSSI, J. P. Rapport de la Commission sur la mesure des performances économiques et du progrès social. 2009.

STOLLE, D.; HOOGHE, M. Inaccurate, exceptional, one-sided or irrelevant? The debate about the alleged decline of social capital and civic engagement in Western societies. British journal of political science, v. 35, n. 1, p. 149-167, 2005.

SVENDSEN, G. T.; SORENSEN, J. F. L. The socioeconomic power of social capital: A double test of Putnam's civic society argument. International Journal of Sociology and Social Policy, v. 26, n. 9/10, p. 411-429, 2006.

TREGEAR, A.; COOPER, S. Embeddedness, social capital and learning in rural areas: The case of producer cooperatives. Journal of Rural Studies, v. 44, n. 1, p. 101-110, 2016.

TSAI, W.; GHOSHAL, S. Social capital and value creation: the role of intrafirm networks. The Academy of Management Journal, v. 41, n. 4, p. 464-476, 1998.

TUNDUI, C.; MACHA, R. Social Capital and Willingness to Pay for Community Based Health Insurance: Empirical Evidence from Rural Tanzania. Journal of Finance and Economics, v. 2, n. 4, p. 50-67, 2014. 
UMANS, L.; ARCE, A. Fixing rural development cooperation? Not in situations involving blurring and fluidity. Journal of Rural Studies, v. 34, p. 337-344, 2014.

WHEELER, S. et al. Handing down the farm? The increasing uncertainty of irrigated farm succession in Australia. Journal of Rural Studies, v. 28, n. 3, p. 266-275, 2012.

WIESINGER, G. The importance of social capital in rural development, networking and decision-making in rural areas. Journal of Alpine Research/ Revue de géographie alpine, n. 95, p. 43-56, 2007.

WIGGINS, S.; KIRSTEN, J.; LLAMBÍ, L. The future of small farms. World development, v. 38, n. 10, p. 1341-1348, 2010.

WOODHOUSE, A. Social capital and economic development in regional Australia: A case study. Journal of Rural Studies, v. 22, n. 1, p. 83-94, 2006.

WOOLCOCK, M.; NARAYAN, D. Social capital: Implications for development theory, research, and policy. The world bank research observer, v. 15, n. 2, p. 225-249, 2000.

YOKOYAMA, M. H.; SEKIGUCHI, T. The use of social network sites in the workplace: A case study in Brazilian companies. Brazilian Business Review, v. 11, n. 2, p. 87, 2014. 